\title{
Resveratrol-based Nutraceuticals for the Management of Diabetes and Obesity: Real Therapeutic Potential or a mere Palliative?
}

\author{
Giuseppe Annunziata*, Gian Carlo Tenore and Ettore Novellino \\ Department of Pharmacy, University of Naples Federico II, Italy
}

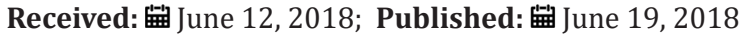

*Corresponding author: Giuseppe Annunziata, Department of Pharmacy, University of Naples Federico II, Via Domenico Montesano 49, 80131, Naples, Italy

\section{Editorial}

It is well known that obesity and diabetes (more specifically, the type 2 diabetes mellitus, T2DM) are currently a growing public health problem, which involves several millions subjects in the World. Years ago, indeed, the term "diabesity" was coined highlighting not only the strong pathogenic relationship between obesity and T2DM, but also the pandemic aspect that link these two diseases. The increasing global prevalence of diabesity represents a grave concern in terms of public health and, at the same time, medical costs, resulting in a serious social economic issue.

Periodically, several scientific societies elaborate guidelines for the management of obesity and T2DM, mainly based on a particular interest in life-style and nutrition, as a useful strategy for prevention and treatment. In addition to the life-style intervention, a large number of drugs are currently available, and some of them (e.g. metformin or incretine-mimetic) are used both in case of diabetes and/or obesity. These pharmacological treatments, however, are not without side-effects. For this reason, the interest in natural drugs is nowadays emerging.

Beside the well-known phytotherapy, based on the use of medical herbs with documented beneficial effects on human health, a consolidated and growing interest is currently in nutraceutical. This term is a crasis between the words "nutrition" and "pharmaceutical", coined in the 1989 by Dr. S. DeFelice. Nutraceutical, thus, is the science studying chemical, composition and medical effects of the food- or animal-derived bioactive compounds, underling the strong influence of the diet on health. Nutraceutical, indeed, is currently recognized to be one the most important natural approach for management (mainly as add-on therapy to the common treatments) and the prevention of several pathologies, acting "beyond diet, before drugs" as stated in 2012 by Prof. E. Novellino [1].
Nowadays a large number of evidence-based data regarding the effects of single or group of food-derived bioactive compounds are available in scientific literature, pointing out the interest of scientific research in nutraceuticals. In particular, several in vitro, animal-based and in vivo studies were carried out demonstrating the effects of nutraceutical in managing obesity and/or diabetes. As an example, we demonstrated that polyphenols from white tea exhibited a strong activity in reducing glucose uptake in HepG2 cell lines [2]. Moreover, it was proposed a possible anti-diabetic or anti-obesity effect of the bitter compounds contained in beer, which may act stimulating the intestinal bitter taste receptors, resulting in increasing the incretine release [3], but further investigation are needed.

Beside this evidence, in this document we would like to focus your attention to the role of resveratrol (RSV) on diabesity. RSV is the most representative polyphenol contained in skin of grapes and wine. Several beneficial effects are attributed to RSV and, among these, the well- and historically-known cardio protection. Interestingly, RSV has been reported to exert anti-diabetes and anti-obesity effects [4]. Although it has not been too much stressed, studies demonstrated an important mechanism of action by which RSV can act in prevention and management of diabesity: the activation of the 5' AMP-activated protein kinase (AMPK) [58], a Ser-Thr kinase strongly involved in regulation of cellular and glucose metabolism [9], target of anti-diabetic drugs, such as metformin $[10,11]$. This AMPK activation is the main target for the RSV metabolic effect [12], resulting in

a) Stimulating the glucose uptake in L6 myotubes $[13,14]$

b) Inhibiting adipocyte differentiation [14]

c) Improving glucose homeostasis and symptoms in animal 
model of gestational diabetes [15]

d) Promoting formation of brown adipocytes and exerting thermogenic effects [16]

With this editorial, we would like to propose a start point for discussion and an input for further investigations about the role of RSV-based nutraceuticals, which have been shown to be not a mere palliative but a novel and promising natural approach for the management of diabesity. We hope this Journal will encourage the scientific discussion regarding the role of nutraceutical sciences in metabolic diseases focusing on the evidences, safety and absence of side-effects, enlarging the knowledge of physician on this issue.

\section{References}

1. Santini A, Tenore GC, Novellino E (2017) Nutraceuticals: A paradigm of proactive medicine. Eur J Pharm Sci 96: 53-61.

2. Tenore GC, Stiuso P, Campiglia P, Novellino E (2013) In vitro hypoglycaemic and hypolipidemic potential of white tea polyphenols. Food Chem 141(3): 2379-2384.

3. Barrea L, Annunziata G, Muscogiuri G, Arnone A, Tenore GC, et al. (2017) Could hop-derived bitter compounds improve glucose homeostasis by stimulating the secretion of GLP-1? Crit Rev Food Sci Nutr 14: 1-8.

4. Szkudelska K, Szkudelski T (2010) Resveratrol, obesity and diabetes. Eur J Pharmacol 635(1-3): 1-8.

5. Baur JA, Pearson KJ, Price NL, Jamieson HA, Lerin C, et al. (2006) Resveratrol improves health and survival of mice on a high-calorie diet. Nature 444(7117): 337-342.

6. Shang J, Chen LL, Xiao FX, Sun H, Ding HC, et al. (2008) Resveratrol improves non-alcoholic fatty liver disease by activating AMP-activated protein kinase. Acta Pharmacol Sin 29(6): 698-706.
7. Rivera L, Morón R, Zarzuelo A, Galisteo M (2009) Long-term resveratrol administration reduces metabolic disturbances and lowers blood pressure in obese Zucker rats. Biochem Pharmacol 77(6): 1053-1063.

8. Penumathsa SV, Thirunavukkarasu M, Zhan L, Maulik G, Menon VP, et al. (2008) Resveratrol enhances GLUT-4 translocation to the caveolar lipid raft fractions through AMPK/Akt/eNOS signalling pathway in diabetic myocardium. J Cell Mol Med 12(6A): 2350-2361.

9. Rutter GA, Da Silva Xavier G, Leclerc I (2003) Roles of 5'-AMP-activated protein kinase (AMPK) in mammalian glucose homoeostasis. Biochem J 375(Pt 1): 1-16.

10. Zhou G, Myers R, Li Y, Chen Y, Shen X, et al. (2001) Role of AMP-activated protein kinase in mechanism of metformin action. Clin Invest 108(8): $1167-1174$.

11. Fryer LG, Parbu Patel A, Carling D (2002) The Anti-diabetic drugs rosiglitazone and metformin stimulate AMP-activated protein kinase through distinct signaling pathways. J Biol Chem 277(28): 25226-25232.

12. Um JH, Park SJ, Kang H, Yang S, Foretz M, et al. (2010) AMP-activated protein kinase-deficient mice are resistant to the metabolic effects of resveratrol. Diabetes 59(3): 554-563.

13. Breen DM, Sanli T, Giacca A, Tsiani E (2008) Stimulation of muscle cell glucose uptake by resveratrol through sirtuins and AMPK. Biochem Biophys Res Commun 374(1): 117-122.

14. Ito Nagahata T, Kurihara C, Hasebe M, Ishii A, Yamashita K, et al. (2013) Stilbene analogs of resveratrol improve insulin resistance through activation of AMPK. Biosci Biotechnol Biochem 77(6): 1229-1235.

15. Yao L, Wan J, Li H, Ding J, Wang Y, et al. (2015) Resveratrol relieves gestational diabetes mellitus in mice through activating AMPK. Reprod Biol Endocrinol 13: 118.

16. Wang S, Liang X, Yang Q Fu X, Zhu M, et al. (2017) Resveratrol enhances brown adipocyte formation and function by activating AMP-activated protein kinase (AMPK) $\alpha 1$ in mice fed high-fat diet. Mol Nutr Food Res 61(4).

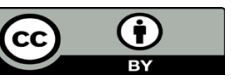

This work is licensed under Creative Commons Attribution 4.0 License

To Submit Your Article Click Here: Submit Article

DOI: $10.32474 / A D 0.2018 .01 .000109$

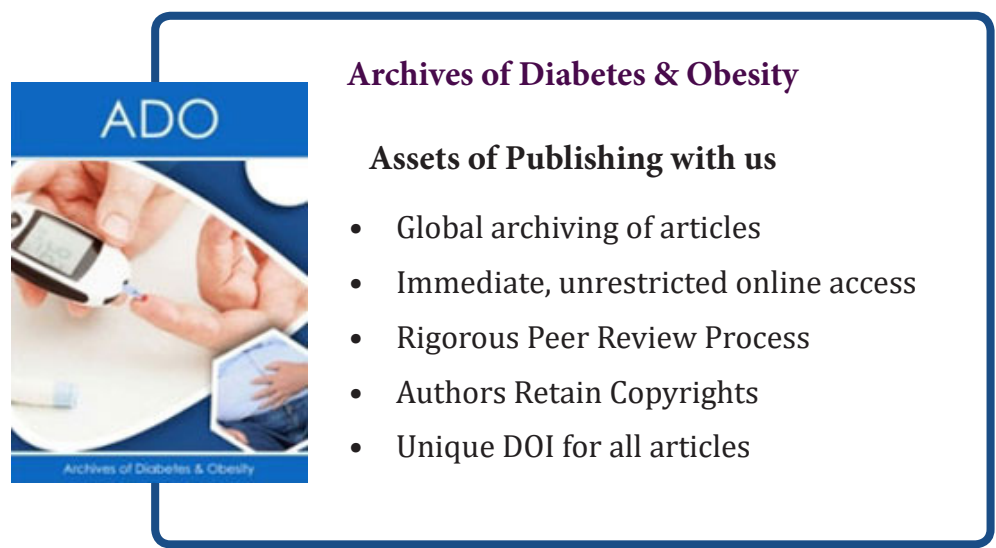

